\title{
ACE-2-interacting Domain of SARS-CoV-2 (AIDS) Peptide Suppresses Inflammation to Reduce Fever and Protect Lungs and Heart in Mice: Implications for COVID-19 Therapy
}

\author{
Ramesh K. Paidi $^{1} \cdot$ Malabendu Jana $^{1} \cdot$ Rama K. Mishra $^{2} \cdot$ Debashis Dutta $^{1} \cdot$ Sumita Raha ${ }^{1} \cdot$ Kalipada Pahan $^{1,3}$ (DD
}

Received: 5 December 2020 / Accepted: 18 December 2020 / Published online: 11 January 2021

(C) The Author(s), under exclusive licence to Springer Science+Business Media, LLC part of Springer Nature 2021

\begin{abstract}
COVID-19 is an infectious respiratory illness caused by the virus strain severe acute respiratory syndrome coronavirus 2 (SARSCoV-2) and until now, there is no effective therapy against COVID-19. Since SARS-CoV-2 binds to angiotensin-converting enzyme 2 (ACE2) for entering into host cells, to target COVID-19 from therapeutic angle, we engineered a hexapeptide corresponding to the ACE2-interacting domain of SARS-CoV-2 (AIDS) that inhibits the association between receptor-binding domain-containing spike S1 and ACE-2. Accordingly, wild type (wt), but not mutated (m), AIDS peptide inhibited SARS-CoV-2 spike S1-induced activation of NF-KB and expression of IL-6 in human lungs cells. Interestingly, intranasal intoxication of C57/ BL6 mice with recombinant SARS-CoV-2 spike S1 led to fever, increase in IL-6 in lungs, infiltration of neutrophils into the lungs, arrhythmias, and impairment in locomotor activities, mimicking some of the important symptoms of COVID-19. However, intranasal treatment with wtAIDS, but not mAIDS, peptide reduced fever, protected lungs, improved heart function, and enhanced locomotor activities in SARS-CoV-2 spike S1-intoxicated mice. Therefore, selective targeting of ACE2-to-SARSCoV-2 interaction by wtAIDS may be beneficial for COVID-19.
\end{abstract}

Keywords COVID-19 · ACE-2 - Spike S1 · Lung inflammation · Fever · Arrhythmias

\section{Introduction}

Common symptoms of COVID-19 are fever, cough, and shortness of breath and with a mortality rate of around 4$5 \%$, it is more than 10 times lethal than the flu. While anyone is susceptible to COVID-19, the ones over 60 or with preexisting conditions, such as hypertension, obesity, asthma, or diabetes, are more vulnerable to severe symptoms (Ledford

Ramesh K. Paidi and Malabendu Jana have equal contribution to the work.

Kalipada Pahan

Kalipada_Pahan@rush.edu

1 Department of Neurological Sciences, Rush University Medical Center, 1735 West Harrison St Suite Cohn 310, Chicago, IL 60612, USA

2 Department of Biochemistry and Molecular Genetics, Feinberg School of Medicine, Northwestern University, Chicago, IL, USA

3 Division of Research and Development, Jesse Brown Veterans Affairs Medical Center, Chicago, IL, USA
2020; Machhi et al. 2020). Until now, no effective therapy is available to tackle this viral pandemic.

Entry of SARS-CoV-2 into the host cells is probably the most important event in COVID-19 disease process. Angiotensin-converting enzyme 2 (ACE2), the main effector of the classical renin-angiotensin system, is a cell surface receptor that is predominant in lung, heart and kidney (Zaman et al. 2002). Although the prototype function of ACE2 is to convert angiotensin II (AngII), a vasoconstrictor, to Ang1-7, a vasodilator, and thereby to play an important role in the pathophysiology of cardiovascular diseases (Vickers et al. 2002; Zaman et al. 2002), recently, ACE2 came to renewed attention due to its requirement by COVID-19 for entering into host cells. It is found that COVID-19 binds to ACE2 via the S protein on its surface (Machhi et al. 2020; Stower 2020). During infection, the $\mathrm{S}$ protein is cleaved into $\mathrm{S} 1$ and $\mathrm{S} 2$ subunits and the S1 subunit encompasses the receptor-binding domain (RBD). Therefore, this subunit permits COVID-19 to directly attach to the peptidase domain of ACE2 (Ledford 2020; Stower 2020). 
Since ACE2 is a beneficial molecule, either inhibiting or knocking down of ACE2 is not a valid option. Therefore, for specific targeting of the binding between ACE2 and SARSCoV-2, we designed a peptide corresponding to the ACE2interacting domain of SARS-CoV-2 (AIDS) that inhibited the binding between ACE2 and SARS-CoV-2 spike S1 and specifically reduced spike S1-mediated activation of NF- $\mathrm{KB}$ and induction of IL-6 in lung cells without modulating doublestranded RNA (poly IC)-, HIV-1 Tat-, and flagellinmediated NF- $\mathrm{kB}$ activation and IL-6 expression. Moreover, intranasal administration of AIDS peptide reduced fever, protected lungs, improved heart function, and enhanced locomotor activities in SARS-CoV-2 spike S1-intoxicated mice, highlighting the therapeutic promise of AIDS peptide in COVID-19.

\section{Materials and Methods}

\section{Reagents}

Human A549 lung carcinoma cell line (cat\# CCL-185) and F12K medium (cat\# 30-2004) were purchased from ATCC. Hank's balanced salt solution, $0.05 \%$ trypsin, and antibioticantimycotic were purchased from Mediatech (Washington, DC). Fetal bovine serum (FBS) was obtained from Atlas Biologicals. ACE2:SARS-CoV-2 Spike Inhibitor Screening Assay Kit (Cat \# 79,936) was purchased from BPS Bioscience. Recombinant COVID-19 Spike protein S1 was purchased from MyBioSource (Cat\# MBS553722) and Abeomics (Cat\# MBS553722). Anti-SARS-CoV-2 Spike S1 antibody (Cat\# A3000-50) was bought from BioVision. Human IL-1 $\beta$ ELISA and IL-6 ELISA kits were bought from ThermoFisher.

\section{Animals and Intranasal Delivery of AIDS Peptides}

Mice were maintained and experiments conducted in accordance with National Institute of Health guidelines and were approved by the Rush University Medical Center IACUC. C57/BL6 mice (6-8 week old; Envigo) of both sexes were treated intranasally with wtAIDS or mAIDS peptides (100 ng/mouse/d) for 7d. Briefly, AIDS peptides were dissolved in $2 \mu \mathrm{l}$ normal saline, mice were hold in supine position and $1 \mu \mathrm{l}$ volume was delivered into each nostril using a pipetman.

\section{Intoxication of C57/BL6 Mice With Recombinant SARS-CoV-2 Spike S1}

C57/BL6 mice (6-8 week old; Envigo) of both sexes were intoxicated with recombinant SARS-CoV-2 spike S1 (50 ng/ mouse/d) intranasally. Briefly, recombinant spike S1 was dissolved in $2 \mu \mathrm{l}$ normal saline, mice were hold in supine position and $1 \mu \mathrm{l}$ volume was delivered into each nostril using a pipetman. Control mice received only $2 \mu \mathrm{l}$ saline.

\section{Non-invasive ECG Recording}

Prior to ECG recording, mice were acclimatized to the ECG pulse transducer pad (AD instruments TN 012/ST, USA) and the experimental housing conditions. ECG pulse transducer pad was placed around the heart of each animal and ECG recording was carried out for $120 \mathrm{~s}$. For ECG analysis, electrocardiography data were exported from the Labchart pro, version 8.0 (Power Lab 4/35 model) as raw data format and the digital signal processing was performed using this software. The recording was conducted for $120 \mathrm{~s}$ and the ECG signals were recorded at a sampling range of $20 \mathrm{mV}$ with 4 beats/s sampling rate as recommended in the software for mouse ECG analysis. The ECG tracing was visually reviewed for identifying possible arrhythmias or other anomalous complexes. Prior to analyze the data, in the Labchart pro software ECG setting data source was kept for ECG channel selected as the channel 1 out of the multiple channels and selected for whole channel specific for mouse species. In the detection setting, typical QRS width was reserved at $10 \mathrm{~ms}$ and $\mathrm{R}$ wave kept at least $60 \mathrm{~ms}$ apart with the alignment maintained at QRS maximum. In the analysis portion, pre-baseline was kept at $10 \mathrm{~ms}$ with maximum at $50 \mathrm{~ms}$. We selected for rodent waves and measured ST segment height at $10 \mathrm{~ms}$ height. All the ECG parameters including PQ interval, QRS interval, QT interval, T duration, JT interval as well as P Amplitude, Q amplitude, R Amplitude, S amplitude, T amplitude were calculated. The recording and analysis settings were kept same for all the experimental mice included in this study.

\section{Monitoring Lung Infiltration and Pathology}

After treatment, animals were anesthetized with ketamine/xylazine injectable followed by transcardial perfusion (Mondal et al. 2018). The lungs were collected and processed for histological studies. Hematoxylineosin (HE) (SigmaAldrich, St Louis, MO) staining was performed from $4 \mu \mathrm{m}$ thick paraffin embedded sections and used for studying the general lung tissue morphology. Number of epithelial cells, and the number infiltrated neutrophils in alveolar spaces and interstitial space were analyzed by NIH Image J. At least ten $40 x$ fields from each group were chosen for the counting of the epithelial and infiltrated neutrophils. Lung injury score was measured as described by Matute-Bello et al. (Matute-Bello et al. 2011) following a scale (Table $\mathrm{S} 1$ ). 


\section{In Silico Structural Analysis}

In silico structural analysis was performed as described earlier (Roy et al. 2015; Rangasamy et al. 2018b). Briefly, by utilizing the protein preparation tools from the Schrodinger, Inc. platform, we evaluated the quality of the crystal structure of human ACE2 and SARS-CoV-2 spike S1. After assessing the quality of the crystal structure, hydrogens were added to the hydrogen bond orientation, charges were added in the Optimized Potential for Liquid Simulations (OPLS3) force field followed by adding missing atoms and side chains of the different residues of both the proteins. Finally, the complex structure was subjected to energy minimization in OPLS3 force field to make it torsion free. After the protein predation, we extracted out the spike protein from the ACE2 and then applied the dynamic hydrogen bonding module in order to find potential hydrogen bonds between the two structures. After assessing the H-bonds, we also evaluated other interactions such as hydrophobic interactions between the two structures as shown in Fig. 1a.

\section{ACE2:SARS-CoV-2 Spike Binding Assay}

The effect of wtAIDS and mAIDS peptides on the binding of ACE2 and SARS-CoV-2 spike was examined using the ACE2:SARS-CoV-2 Spike inhibitor screening assay kit (BPS Bioscience, San Diego, CA) according to manufacturer's instructions. Briefly, 96-well nickel-treated plate provided by the manufacturer was coated with ACE2 solution. After washing with immuno buffer and incubation with blocking buffer, different concentrations of AIDS peptides were added to each well followed by addition of SARSCoV-2 Spike (RBD)-Fc. After washing and incubation with blocking buffer, plates were treated with anti-mouse Fc-HRP followed by addition of an HRP substrate. Resultant chemiluminescence was measured using Perkin Elmer multimode microplate reader, Victor X5.

\section{Semi-quantitative RT-PCR Analysis}

Total RNA was isolated from A549 lung cells and mouse lungs using RNAeasy Mini kit (Qiagen, Germantown, MD) and Ultraspec-II RNA reagent (Biotecx Laboratories, Inc., Houston, TX), respectively. To remove any contaminating genomic DNA, total RNA was digested with DNase. RTPCR was carried out as described earlier (Ghosh et al. 2007; Roy et al. 2013) using a RT-PCR kit (Clontech, Mountain View, CA). Amplified products were electrophoresed on a $1.8 \%$ agarose gels and visualized by ethidium bromide staining. Message for the GAPDH gene was used to ascertain that an equivalent amount of cDNA was synthesized from different samples.

\section{Real-time PCR Analysis}

DNase-digested RNA was analyzed by real-time PCR in the ABI-Prism7700 sequence detection system (Applied Biosystems, Foster City, CA) as described earlier (Ghosh et al. 2007; Roy et al. 2013).

\section{EMSA}

Nuclear extracts were prepared, and EMSA was performed as described previously (Pahan et al. 2001; Rangasamy et al. 2018b) with minor modifications. Briefly, IRDye infrared dye end-labeled oligonucleotides containing the consensus binding sequence for NF- $\mathrm{KB}$ were purchased from Licor Biosciences. Six micrograms of nuclear extract was incubated with binding buffer and with infrared-labeled probe for $20 \mathrm{~min}$. Subsequently, samples were separated on a $6 \%$ polyacrylamide gel in $0.25 \times$ TBE buffer (Tris borate-EDTA) and analyzed by the Odyssey Infrared Imaging System (LI-COR Biosciences).

\section{In situ ChIP}

Recruitment of NF- $\mathrm{KB}$ to the $I L-6$ promoter in vivo in the lung of mice was examined by in situ ChIP analysis as described before (Roy et al. 2013). Briefly, after fixation in formaldehyde, lungs were kept in $4 \%$ paraformaldehyde for overnight followed by washing with PBS and then homogenization in Tris-EDTA buffer ( $\mathrm{pH}$ 7.6). The homogenates were kept in $500 \mu \mathrm{L}$ lysis buffer at $52{ }^{\circ} \mathrm{C}$ for overnight until tissue fragments were dissolved completely. After that, the genomic DNA was isolated and sonicated followed by immunoprecipitation with antibodies against $\mathrm{p} 65, \mathrm{p} 50, \mathrm{p} 300$, and RNA polymerase according to standard protocol as described by us (Jana et al. 2007; Rangasamy et al. 2018a). Control IgG was also run in parallel. Immunoprecipitated DNA was analyzed by PCR and real-time PCR using following primers:

\section{Sense 5'-CCAATCAGCCCCACCCACTCTGGC CCC-3'. \\ Anti-sense 5'-GGAATTGACTATCGTTCTTG GTGGGCT-3'.}

\section{ELISA for IL-6 and C-reactive Protein (CRP)}

IL-6 ELISA was performed in lung homogenates and mouse serum as described earlier (Mondal et al. 2020) using an assay kit (eBioscience) according to manufacturer's instruction. CRP ELISA was performed in mouse serum using a kit from Abcam. 
a

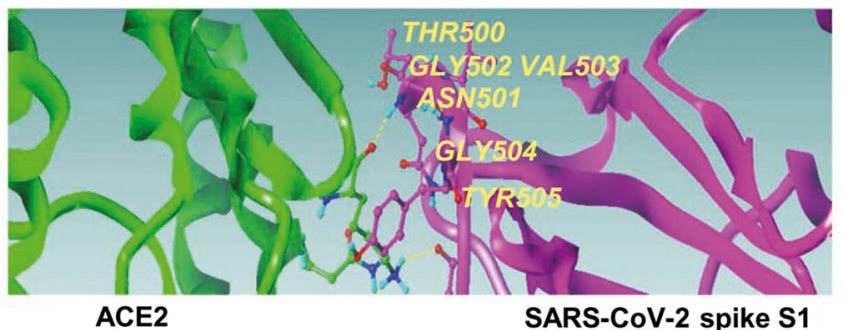

C

b AIDS peptides

wtAIDS: ${ }^{500}$ TNGVG $\underline{Y}^{505}$

mAIDS: ${ }^{500} \underline{T G G V G}^{505}$

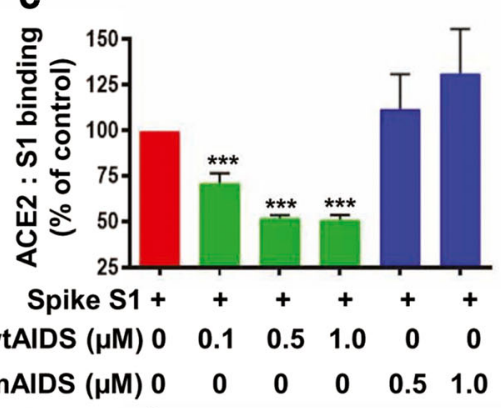

h
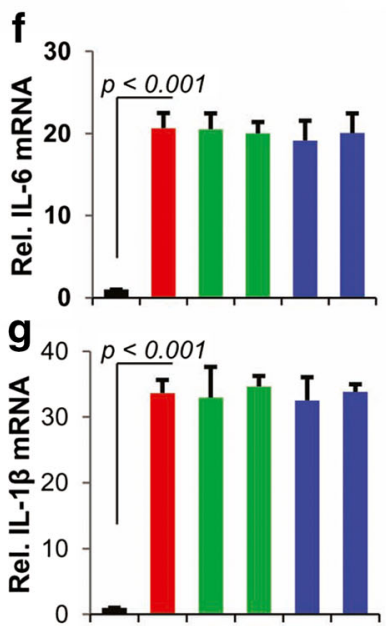

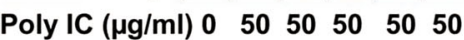

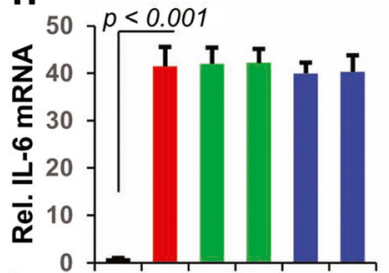

i 125

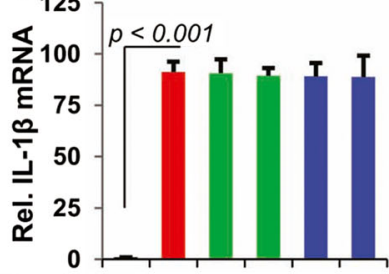

Tat (150 ng/ml) - + + + +

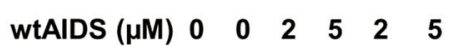
mAIDS ( $\mu$ M) $00 \begin{array}{lllll}0 & 0 & 0 & 2 & 5\end{array}$

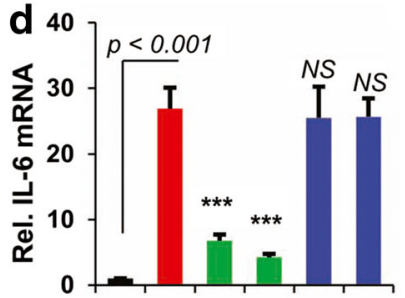

e 50$]^{p<0.001}$

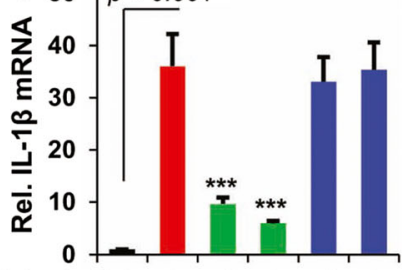

Spike S1 (ng/ml) $0 \begin{array}{llllll}1 & 1 & 1 & 1 & 1\end{array}$

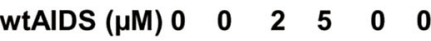

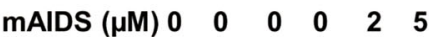
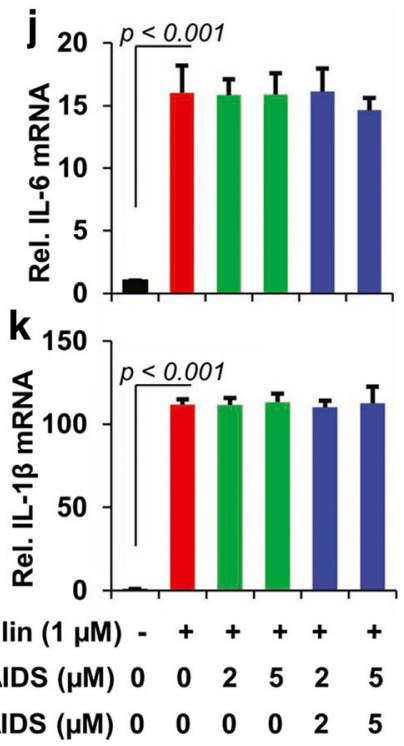

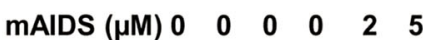

I

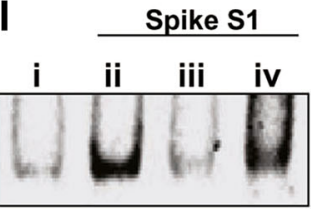

(i) Control m Poly IC

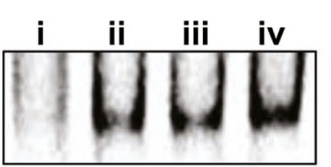

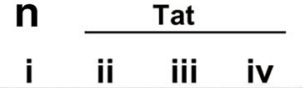

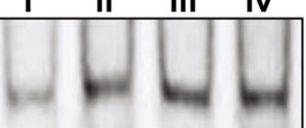

- Flagellin

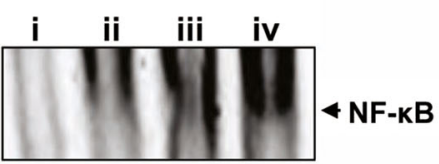

Fig. 1 Designing a peptide for disruption of ACE2 and SARS-CoV-2 interaction. a A rigid-body in silico docked pose of human ACE2 (green) and SARS-CoV-2 spike S1 (magenta). b Sequence of wild type and mutated ACE2-interacting domain of SARS-CoV-2 (AIDS) peptides. Positions of mutations are underlined. $\mathbf{c}$ Inhibition of ACE2 to SARSCoV-2 spike S1 binding by wtAIDS, but not mAIDS, peptide. ***p $<$ 0.001 vs. spike S1. Human A549 lung cells pretreated with different concentrations of wtAIDS and mAIDS peptides for $15 \mathrm{~min}$ were stimulated with $1 \mathrm{ng} / \mathrm{ml}$ recombinant SARS-CoV-2 spike S1 under serum-free condition for $4 \mathrm{~h}$ followed by monitoring the mRNA expression of IL-6 (d) and IL-1 $\beta$ (e) by real-time PCR. Similarly, the effect of wtAIDS and mAIDS peptides on the mRNA expression of IL- $6(\mathbf{f}, \mathbf{h} \& \mathbf{j})$ and IL-1 $\beta$ (g, $\mathbf{i} \& \mathbf{k}$ ) was examined in polyIC- (f \& $\mathbf{g}), \mathrm{HIV}-1$ Tat- (H \& I) and flagellin(j \& k) stimulated A549 cells by real-time PCR. ***p $<0.001$ vs. spike S1. Similarly, the effect of wtAIDS and mAIDS peptides on the activation of NF-kB was examined in spike S1- (l), polyIC- (m), HIV-1 Tat- (n), and flagellin- (o) stimulated A549 cells by EMSA. In this case, cells were stimulated with spike S1, polyIC, HIV-1 Tat, and flagellin for $1 \mathrm{~h}$. Results represent three independent experiments 


\section{Statistical Analysis}

Statistical analyses were performed using GraphPad Prism 8.0 (GraphPad Software, Inc., La Jolla, CA). Mouse behavioral measures were examined by an independent one-way ANOVA using SPSS. Homogeneity of variance between test groups was examined using Levene's test. Post-hoc analyses were conducted using Tukey's tests. Other data were expressed as means $\pm \mathrm{SD}$ of three independent experiments. Statistical differences between means were calculated by the Student's $t$-test (two-tailed). A $p$-value of less than 0.05 ( $\mathrm{p}<$ $0.05)$ was considered statistically significant.

\section{Results}

Designing of a Peptide Corresponding to the ACE2interacting Domain of SARS-CoV-2 (AIDS) Since there is no specific treatment for COVID-19, from the therapeutic angle, we decided to target the interaction between SARS-CoV-2 and its receptor ACE2. The receptor-binding domain (RBD) of SARS-CoV-2 spike S1 is involved in the interaction with ACE2 (Du et al. 2009). Therefore, we applied rigid-body protein-protein interaction tool to model the interaction between RBD of spike protein S1 subunit and ACE2. As evident from our in silico modeling analysis, the docked pose of ACE2 and S1 RBD complex revealed a strong H-bond between Asn501 of spike S1 and Lys353 of ACE2. In addition, Tyr505 of spike S1 exhibited a hydrophobic interaction with Gln42 and the side chain of Lys353 of ACE2 (Fig. 1a). Therefore, we designed a hexapeptide (Fig. 1b) corresponding to the ACE2interacting domain of SARS-CoV-2 (AIDS) from the RBD of $\mathrm{S} 1$ subunit to unsettle the interaction between SARS-CoV-2 and ACE2.

\section{Wild type (wt) AIDS: ${ }^{500}$ TNGVGY ${ }^{505}$ \\ Mutated (m) AIDS: ${ }^{500}$ TG $\overline{G V G D} \underline{5}^{\overline{505}}$}

Positions of mutations are underlined. Next, to examine whether wtAIDS peptide inhibits the binding of ACE2 with SARS-CoV-2 spike S1, we employed chemiluminescencebased ACE2:SARS-CoV-2 spike S1 binding using an assay kit (catalog\# 79,936; BPS Bioscience). As evident from Fig. 1c, SARS-CoV-2 spike S1 binding to immobilized ACE2 was strongly inhibited by wtAIDS peptide. However, no such inhibition was found with mAIDS peptide (Fig. 1c), indicating the specificity of the effect.

AIDS Peptide Inhibits Lung Cell Inflammation Induced By SARS-CoV-2 Spike S1, But Not Double-stranded RNA (poly IC), HIV-1 Tat, and Bacterial Flagellin Pulmonary inflammation ultimately leading to acute lung injury is becoming a hallmark of COVID-19 patients visiting ICU (Pia 2020). In addition to
COVID-19, pulmonary complications are also evident in different bacterial and viral infections (Edwards et al. 2012; McCullers 2014). Therefore, we investigated if AIDS peptide was capable of suppressing the expression of proinflammatory molecules in human A549 lung cells induced by different stimuli. A549 cells pretreated with different concentrations of wtAIDS and mAIDS peptides for $15 \mathrm{~min}$ were stimulated with recombinant SARS-CoV-2 spike S1, poly IC, HIV-1 Tat, and bacterial flagellin. At first, we examined whether recombinant SARS-CoV-2 spike S1 was capable of inducing proinflammatory cytokines in A549 lung cells. Dose-dependent analysis showed that SARS-CoV-2 spike S1 was very potent in inducing proinflammatory cytokines and that spike $\mathrm{S} 1$ even at a dose of $0.2 \mathrm{ng} / \mathrm{ml}$ significantly induced the mRNA expression of IL- 6 and IL-1 $\beta$ in lung cells with maximum induction at $1 \mathrm{ng} / \mathrm{ml}$ (Fig. S1A-C). Inability of boiled recombinant SARS-CoV-2 spike S1 to induce the expression of IL-6 and IL-1 $\beta$ in A549 cells (Fig. S1A-C) and neutralization of SARS-CoV-2 spike S1-mediated expression of these cytokines by anti-SARS-CoV-2 spike S1 antibody (Fig. S2A-C) suggest that the induction of proinflammatory molecules in lung cells is due to SARS-CoV-2 spike S1 protein. Moreover, these results also suggest that the so-called "cytokine storm" observed in some COVID-19 patients may be due to the function of spike S1.

Similarly, poly IC (Fig. 1f-g \& Fig. S3B), HIV-1 Tat (Fig. 1h-i \& Fig. S3C), and flagellin (Fig. 1j-k \& Fig. S3D) also increased the expression of IL- 6 and IL- $1 \beta$ in A549 cells. However, wtAIDS peptides inhibited SARS-CoV-2 spike S1mediated induction of IL- 6 and IL-1 $\beta$ in A549 cells (Fig. 1de \& Fig. S3A). In contrast, wtAIDS peptides remained unable to decrease the expression of IL- 6 and IL- $1 \beta$ induced by poly IC (Fig. 1f-g \& Fig. S3B), HIV-1 Tat (Fig. 1h-i \& Fig. S3C), and flagellin (Fig. 1j-k \& Fig. S3D). These results were specific as mAIDS peptides had no effect on the expression of IL6 and IL- $\beta$ induced by any of the stimuli used.

Since activation of NF- $\mathrm{kB}$ plays an important role in the expression of different proinflammatory molecules, we also examined the effect of AIDS peptides on the activation of $\mathrm{NF}-\mathrm{KB}$ in A549 cells. Consistent to the inhibition of spike S1-mediated expression of proinflammatory molecules, wtAIDS, but not mAIDS, peptide suppressed the activation of NF-KB in spike S1-stimulated A549 cells (Fig. 11). On the other hand, either wtAIDS or mAIDS peptide had no effect on the activation of NF- $\mathrm{KB}$ in A549 cells induced by poly IC (Fig. 1m), HIV-1 Tat (Fig. 1n), and flagellin (Fig. 1o), indicating the specificity.

Intranasal Administration of SARS-CoV-2 Spike S1 Causes Lung Inflammation and Fever: Protection By WtAIDS Peptide Similar to that seen in human lung cells, intranasal intoxication of SARS-CoV-2 spike S1 (Fig. 2a) induced the activation of NF-KB in vivo in the lung of C57/BL6 mice 


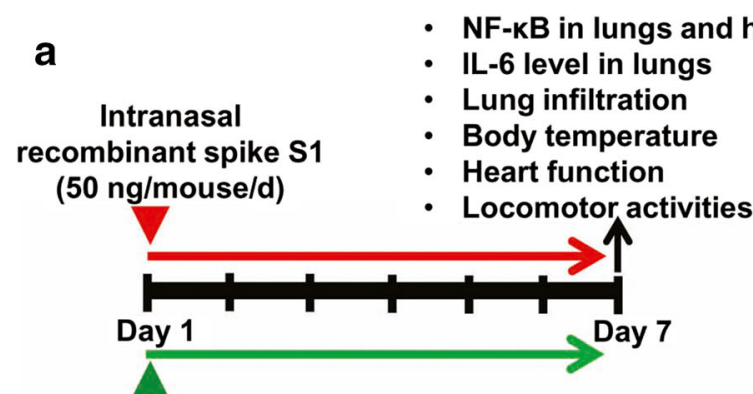

Intranasal AIDS peptides

(100 ng/mouse/d)
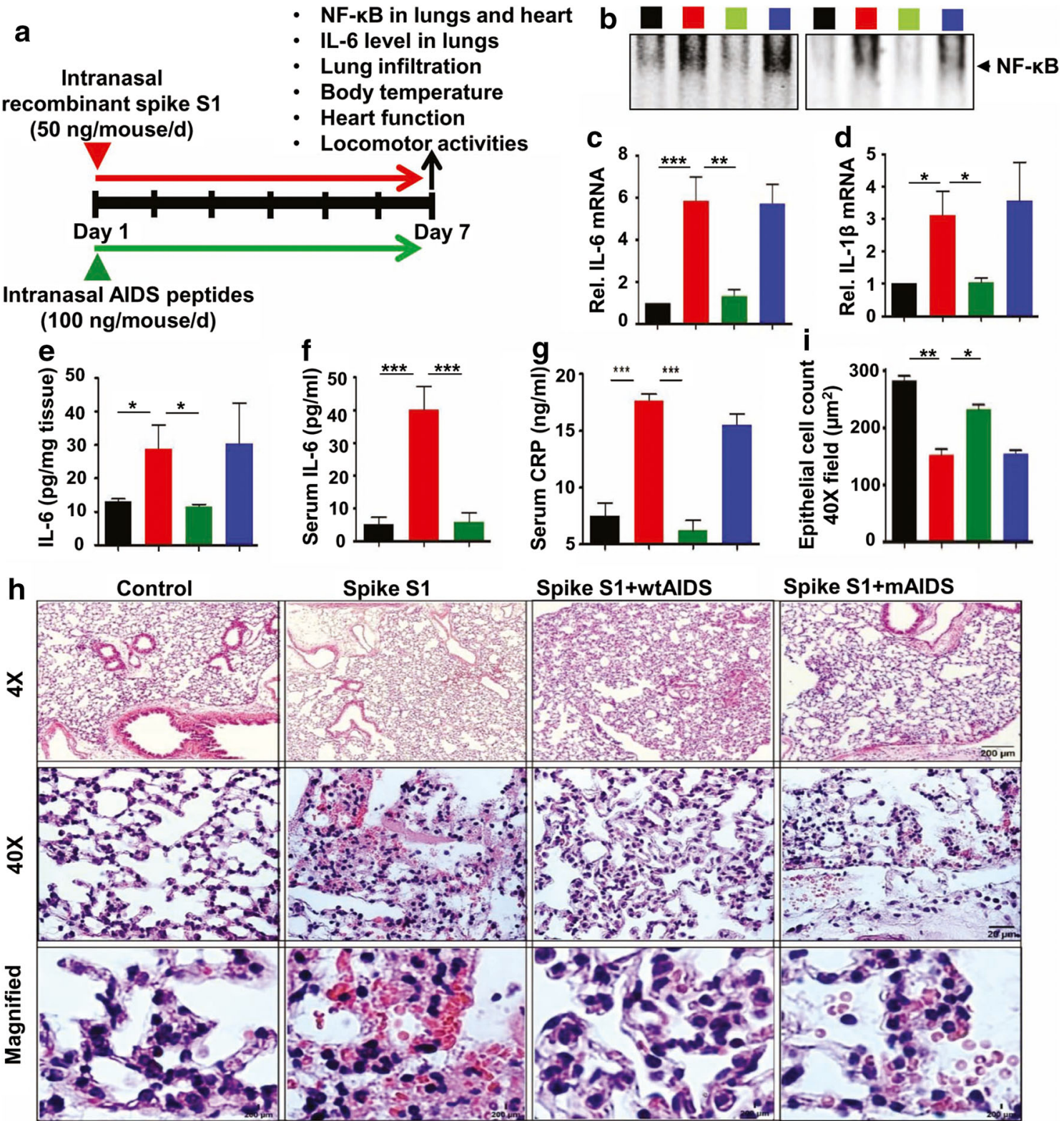

$\square$ Control $\square$ Spike S1

Spike S1 + wtAIDS

Spike S1 + mAIDS
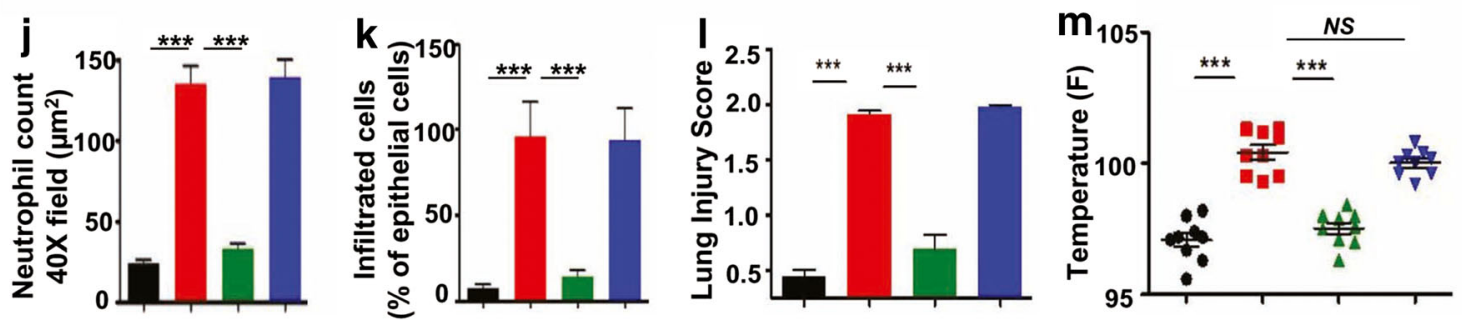

Fig. 2 Intranasal delivery of wtAIDS peptide decreases lung infiltration and inflammation and reduces fever in a mouse model of COVID-19. Six-eight week old C57/BL6 mice $(n=9)$ of both sexes were treated intranasally with wtAIDS or mAIDS peptides (100 ng/mouse/d). After $10 \mathrm{~min}$, mice were intoxicated with recombinant SARS-CoV-2 spike S1 $(50 \mathrm{ng} / \mathrm{mouse} / \mathrm{d})$ via intranasal route. a Schematic presentation of experiments. After $7 \mathrm{~d}$ of treatment, the activation of NF-KB was checked in lung tissues by EMSA (b) followed by monitoring the mRNA expression of IL-6 (c) and IL-1 $\beta$ (d) in lung by real-time PCR. IL-6 protein was measured in lung tissue homogenates by ELISA (e). Levels of IL-6 (f) and CRP $(\mathbf{g})$ were also quantified in serum by ELISA. Lung sections were analyzed by H\&E (h, images of different magnification; $\mathbf{i}$, epithelial cell count; $\mathbf{j}$, neutrophil cell count; $\mathbf{k}$, infiltrated cells as percent of epithelial cells; $\mathbf{l}$, lung injury score). Cells were counted from two sections of each of five mice $(n=5)$ per group. Body temperature $(\mathbf{m})$ was monitored by Cardinal Health Dual Scale digital rectal thermometer. Results are mean \pm SEM of nine mice per group. ${ }^{*} p<0.05 ;{ }^{* *} p<0.01 ;{ }^{* * *} p<0.001$ 
(Fig. 2b). In situ ChIP assay of lung indicated the recruitment of NF- $\kappa$ B subunits p 65 and p50 as well as histone acetyltransferase p300 to the $I L-6$ gene promoter in vivo in the lung of SARS-CoV-2 spike S1-intoxicated mice (Fig. 3a-e. Consistently, SARS-CoV-2 spike S1 was also able to recruit RNA polymerase to the $I L-6$ gene promoter in vivo in the lung (Fig. 3f). These results are specific as no product amplification was observed in immunoprecipitates with control IgG (Fig. 3g). Therefore, SARS-CoV-2 spike S1 intoxication induces the transcription of IL-6 gene in vivo in the lung via NF- $\kappa \mathrm{B}$ activation. Accordingly, we found marked increase in IL-6 mRNA (Fig. 2c) and protein (Fig. 2e) as well as another proinflammatory cytokine (IL-1 $\beta$ ) mRNA (Fig. 2d) in lungs of SARS-CoV-2 spike S1-intoxicated mice as compared to control mice receiving only saline. SARS-CoV-2 spike S1 intoxication also increased the level of IL-6 in serum (Fig. 2f). These results are consistent with the finding that in some COVID-19 patients, disease progression leads to "cytokine storm" and that among these cytokines, IL-6 plays an important role as elevated levels of IL-6 closely correlates to critical illness (Costela-Ruiz et al. 2020). It has been suggested that C-reactive protein (CRP) could be a promising biomarker for assessing the lethality of COVID-19 (Sahu et al. 2020). Accordingly, we noticed marked upregulation of CRP in serum of SARS-CoV-2 spike S1-intoxicated mice (Fig. 2g).

Since shortness of breath is an important issue of COVID-19 patients in the ICU (Chand et al. 2020), we also examined if intranasal administration of SARS-CoV2 spike $\mathrm{S} 1$ could mimic some of the pulmonary features of COVID-19. We found widespread infiltration of neutrophils into the lungs of SARS-CoV-2 spike S1intoxicated mice as compared to control mice receiving only saline (Fig. 2h). Cell counting as well as assessment of lung injury using a scale (Matute-Bello et al. 2011) (Table S1) indicated a loss of lung epithelial cells (Fig. 2i), a marked increase in lung neutrophil infiltration (Fig. 2j-k) and an overall increase in lung injury (Fig. 21) after SARS-CoV-2 spike S1-intoxication. One of the most common symptoms of COVID-19 is fever (Machhi et al. 2020; Pahan and Pahan 2020). Interestingly, daily intranasal administration of SARS-CoV-2 spike S1 at a very low dose (Fig. 2a) led to increase in body temperature (Fig. 2m). However, intranasal treatment of wtAIDS peptide inhibited lung activation of NF-KB (Fig. 2b),
Fig. 3 SARS-CoV-2 spike S1 intoxication induces the recruitment of NF-KB to the $\boldsymbol{I L}-\boldsymbol{G}$ gene promoter in vivo in the lungs: Suppression by wtAIDS treatment. a The map of mouse IL-6 promoter region that harbors one consensus NF- $\mathrm{kB}$-binding site (position - 124 to - 110). Sixeight week old C57/BL6 mice of both sexes were treated intranasally with wtAIDS or mAIDS peptides (100 ng/mouse/d). After $10 \mathrm{~min}$, mice were intoxicated with recombinant SARS-CoV-2 spike S1 (50 ng/mouse/d) via intranasal route. After $7 \mathrm{~d}$ of treatment, in situ ChIP for p65 and p50 followed by semiquantitative (b) and quantitative PCR (c, p65; d, p50; e, p300; f, RNA polymerase; g, control IgG) analyses of $I L-6$ promoter were performed. Results are mean + SEM of four mice per group. ${ }^{* * * *} p<0.001$. h A schema depicting spike S1-induced transcriptional activation of the $I L-6$ gene
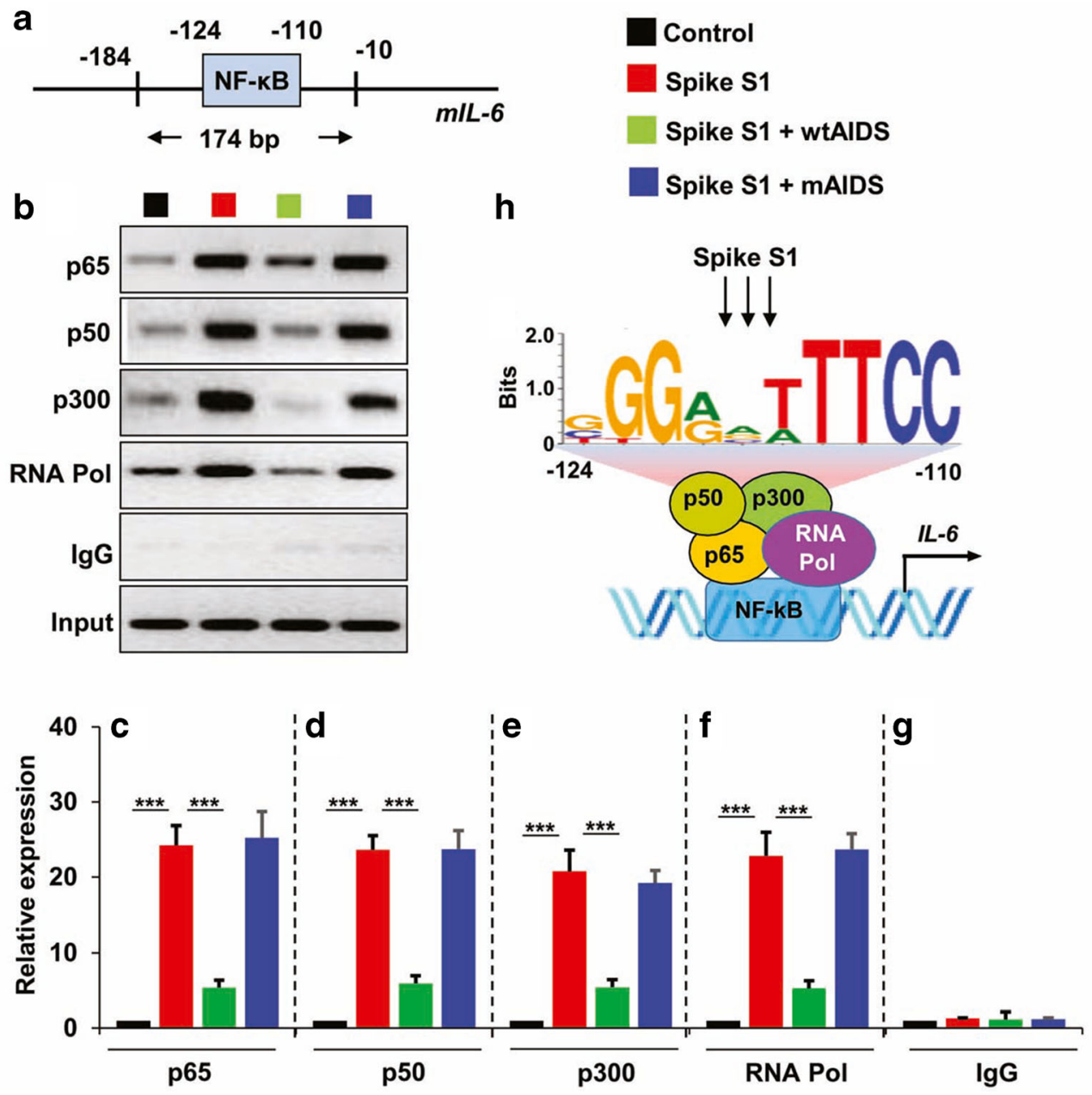
suppressed the recruitment of NF- $\mathrm{B}$ p 65 , NF- $\mathrm{kB}$ p 50 , p300, \& RNA polymerase to $I L-6$ gene promoter in vivo in the lung (Fig. 3), decreased the level of $I L-6$ mRNA and protein as well as $I L-1 \beta$ mRNA in lungs (Fig. $2 \mathrm{c}-\mathrm{e}$ ), lowered the serum levels of IL-6 (Fig. 2f) and CRP (Fig. 2g), reduced lung injury (Fig. 2h-1), and normalized body temperature (Fig. $2 \mathrm{~m}$ ) in SARS-CoV-2 spike S1intoxicated mice. These results were specific as mAIDS peptide had no such inhibitory effect (Figs. 2 and 3).

\section{The WtAIDS Peptide Recovers Heart Functions and Improves} Locomotor Activities in SARS-CoV-2 Spike S1-intoxicated Mice Since prolonged activation of NF-KB appears to be detrimental and promotes heart failure (Gordon et al. 2011), we monitored NF- $\mathrm{kB}$ activation in heart. Similar to that seen in human lung cells (Fig. 11) and mouse lung (Fig. 2b), SARS-CoV-2 spike $\mathrm{S} 1$ insult increased the activation of NF- $\mathrm{KB}$ in vivo in the heart of C57/BL6 mice (Fig. 4a). Many COVID-19 patients in the ICU develop cardiac arrhythmias (Karamchandani et al. 2020). Therefore, we examined if these cardiac features of COVID-19 could be modeled in SARS-CoV-2 spike S1intoxicated mice. Different cardiac parameters are schematically presented in Figure S4. Spike S1-intoxication led to cardiac arrhythmias in mice as indicated by non-invasive ECG (Fig. 4b-c), an increase in heart rate (Fig. 4f), RR interval (Fig. 4g), JT interval (Fig. 4h), and R amplitude (Fig. 4i) and decrease in heart rate variability (Fig. 4j), QRS interval (Fig. 4k) and QT interval (Fig. 4l). Moreover, serum LDH level was markedly higher in Spike S1-intoxicated mice than normal mice receiving saline (Fig. $4 \mathrm{~m}$ ). However, treatment with wtAIDS, but not mAIDS, peptide led to suppression of NF-kB activation (Fig. 4a), normalization of ECG (Fig. 4b-e), stabilization of heart rate (Fig. 4f), RR interval (Fig. 4g), JT interval (Fig. 4h), R amplitude (Fig. 4i), heart rate variability (Fig. 4j), QRS interval (Fig. 4k), and QT interval (Fig. 4l) and regularization of serum LDH (Fig. $4 \mathrm{~m}$ ) in SARS-CoV-2 spike S1-intoxicated mice.

Next, to examine whether spike S1 intoxication also caused functional deficits, we monitored locomotor and open-field activities. Spike S1 insult decreased overall locomotor activities as evident by heat map (Fig. S5A), distance travelled (Fig. S5B), velocity (Fig. S5C), cumulative duration (Fig. S5D), and rotorod performance (Fig. S5E). Similar to normalization of heart functions, wtAIDS, but not mAIDS, peptide also improved SARS-CoV-2 spike S1-induced hypolocomotion (Fig. S5).

\section{Does wtAIDS Peptide Halt the Disease Progression? COVID-} 19 patients are treated with drugs usually after the diagnosis of the disease. Therefore, we investigated whether wtAIDS administered $1 \mathrm{~d}$ after initiation of the disease (Fig. 5a) was still capable of protecting mice from COVID-19 related complications. As evident from body temperature (Fig. 5b), nasal
wtAIDS treatment significantly reduced fever. Similarly, assay of cardiac features by non-invasive ECG showed that spike S1-intoxicated mice receiving wtAIDS from $1 \mathrm{~d}$ after the initiation of the disease displayed very little cardiac arrhythmias (Fig. 5c-e), stabilization of heart rate (Fig. 5f) and RR interval (Fig. 5g), and normalization of heart rate variability (Fig. 5h) and QRS interval (Fig. 5i). Accordingly, wtAIDS peptide administered from the treatment mode also improved locomor activities as evident by heat map (Fig. S6A), distance travelled (Fig. S6B), velocity (Fig. S6C), cumulative duration (Fig. S6D), and rotorod performance (Fig. S6E). These results suggest that wtAIDS peptide is capable of slowing down the disease progression in a mouse model.

\section{Discussion}

Until now, more than 1.5 million people died throughout the world due to COVID-19. Therefore, untangling the mechanism of the disease process of COVID-19 and designing an effective therapeutic approach to slow down the disease and stop the death are of paramount importance. ACE2 being the main player of the classical renin-angiotensin pathway plays an important role in vascular diseases (Zaman et al. 2002). Since SARS-CoV-2 binds ACE2 and causes its internalization to enter into human cells, COVID-19 is particularly deleterious to patients with underlying cardiovascular issues. Although ACE2 inhibitors are available (Jiang et al. 2014) and such inhibitors may stop the entry of SARS-CoV-2 into human cells, it is not possible to inhibit this beneficial molecule. Therefore, through structural analysis of the interaction between SARS-CoV-2 and ACE2, we have designed a small hexapeptide corresponding to the ACE2-interacting domain of SARS-CoV-2 (AIDS). Since the spike S1 of SARS-CoV2 interacts with ACE2, wtAIDS peptide unsettled the association between ACE2 and SARS-CoV-2 spike S1.

Although it has been shown that a subgroup of patients with severe COVID-19 symptoms suffer from cytokine storm (Mehta et al. 2020), underlying mechanism was not known. Marked induction of proinflammatory cytokines (IL-6 and IL$1 \beta$ ) in human A549 lung cells by SARS-CoV-2 spike S1 even at a very low dose suggests that this spike subunit may contribute to cytokine storm in COVID-19 patients. Consistent to the inhibition of association between ACE2 and SARS-CoV2 spike S1, wtAIDS peptide inhibited the activation of NF- KB and the expression of IL- 6 and IL- $1 \beta$ in SARS-CoV- 2 spike S1-intoxicated A549 lung cells. In contrast, wtAIDS peptide did not inhibit the activation of NF- $\mathrm{KB}$ and the expression of proinflammatory cytokines induced by poly IC (viral doublestranded RNA mimic), Tat (transactivator of HIV-1 transcription) and flagellin (a component of bacterial infection), indicating the selective nature of wtAIDS peptide. Most 


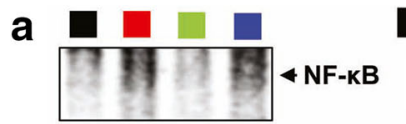

$\square$ Control $\square$ Spike S1 Spike S1+wtAIDS $\square$ Spike S1+mAIDS
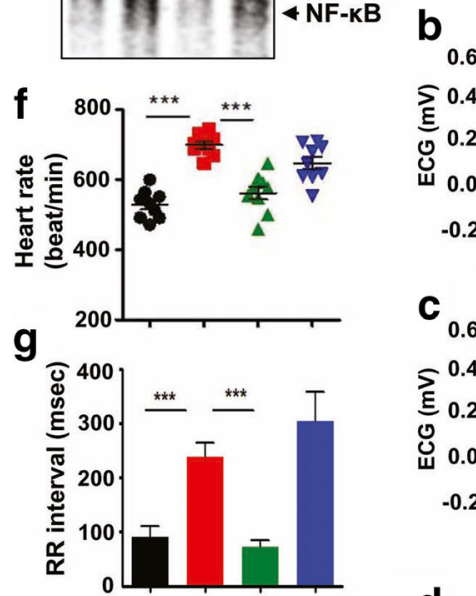

Control

h
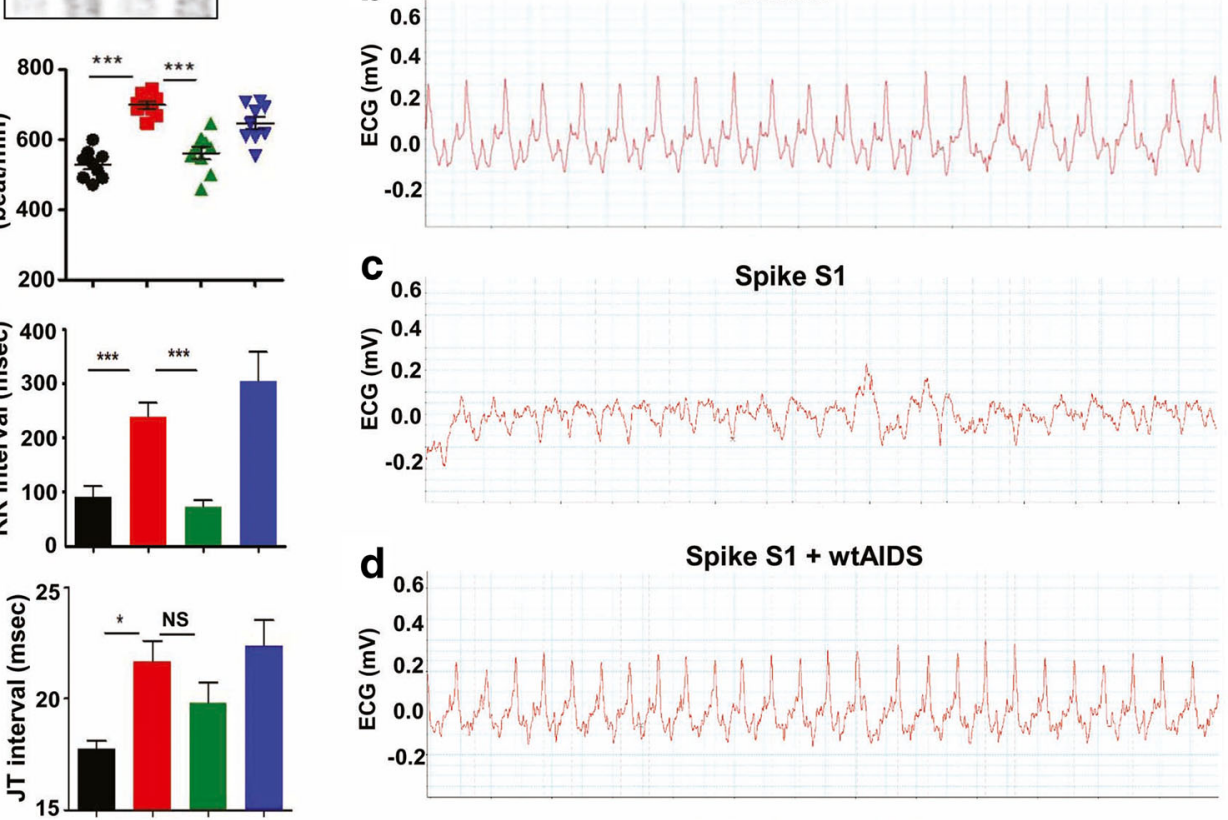

Co.6 Spike S1

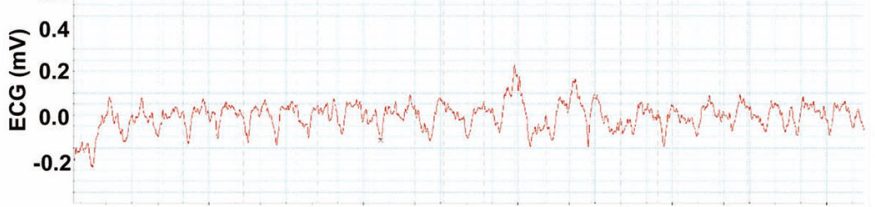

d $_{0.6} \quad$ Spike S1 + wtAIDS

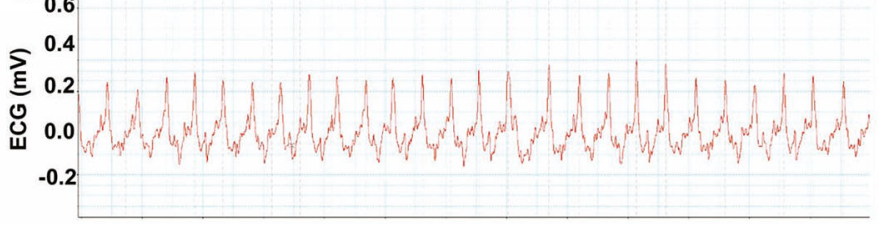

i

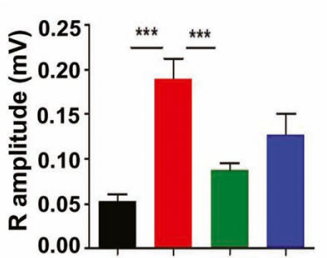

$\mathbf{e}_{0.6}$

Spike S1 + mAIDS
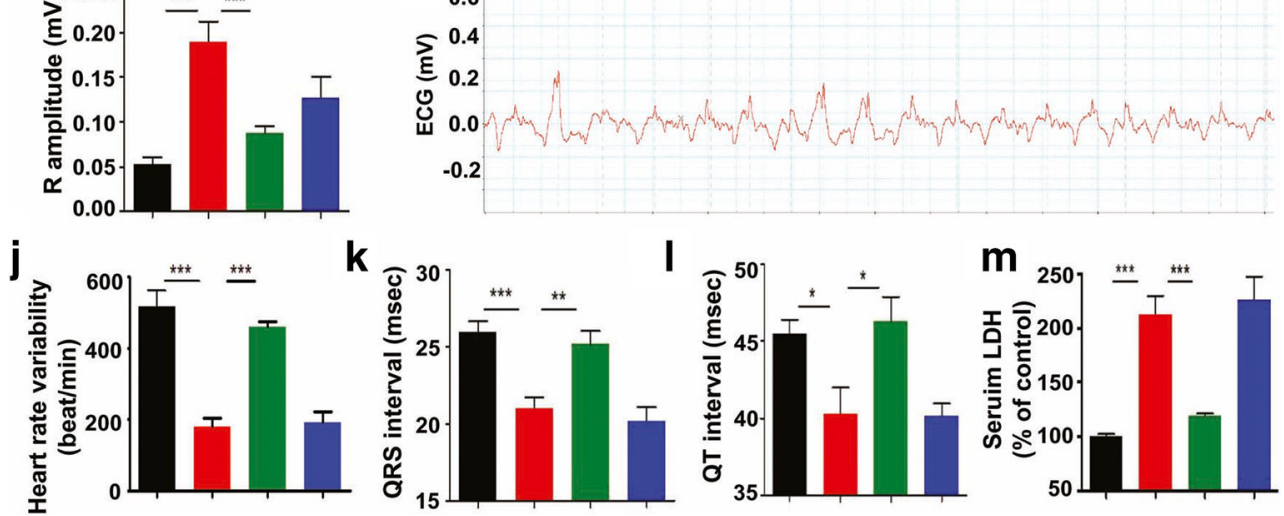

Fig. 4 Intranasal delivery of wtAIDS peptide protects heart functions in a mouse model of COVID-19. Six-eight week old C57/BL6 mice $(n=9)$ of both sexes were treated intranasally with wtAIDS or mAIDS peptides $(100 \mathrm{ng} / \mathrm{mouse} / \mathrm{d})$. After $10 \mathrm{~min}$, mice were intoxicated with recombinant SARS-CoV-2 spike S1 (50 ng/mouse/d) via intranasal route. After 7d of treatment, the activation of NF- $\mathrm{KB}$ was checked in the heart by EMSA (a) followed by monitoring heart functions by non-invasive electrocardiography (ECG) using the PowerLab (ADInstruments) $[\mathbf{b}$, chromatogram of

control mice; c, chromatogram of spike S1-intoxicated mice; d, chromatogram of (spike S1 + wtAIDS)-treated mice; e, chromatogram of (spike S1 + mAIDS)-treated mice; f, heart rate; g, RR interval; h, JT interval; i, R amplitude; $\mathbf{j}$, heart rate variability; $\mathbf{k}$, QRS interval; l, QT interval]. m) Serum LDH was quantified using an assay kit from Sigma. Results are mean + SEM of nine mice per group. ${ }^{*} p<0.05 ;{ }^{* *} p<0.01$; ${ }^{* * * *} p<0.001 ; \mathrm{NS}$, not significant

importantly, the beauty of our finding is that wtAIDS peptide corresponds to peptide sequence of SARS-CoV-2. Therefore, it will only inhibit the binding of SARS-CoV-2 with ACE2 without affecting basal level and beneficial functions of ACE2. Moreover, it will function only in the presence of SARS-CoV-2.

Developing a small animal model system is an important step in understanding mechanisms associated to deadly cardiovascular and pulmonary issues of COVID-19 and evaluating effective drugs for this global pandemic. In normal mice,
SARS-CoV-2 does not easily bind to ACE2, making it difficult to study the course of infection. It has been shown that SARS-CoV-2 infects transgenic mice expressing human ACE2 (Bao et al. 2020). Although regular SARS-CoV-2 does not infect BALB/c mice, N501Y mutated SARS-CoV-2 readily infects BALB/c mice (Gu et al. 2020). However, these mouse models do not display fever and cardiac problems (arrhythmias), two important characteristics of COVID-19. Therefore, drugs generated from these mouse models may not be effective for severe COVID-19 patients in the ICU. 

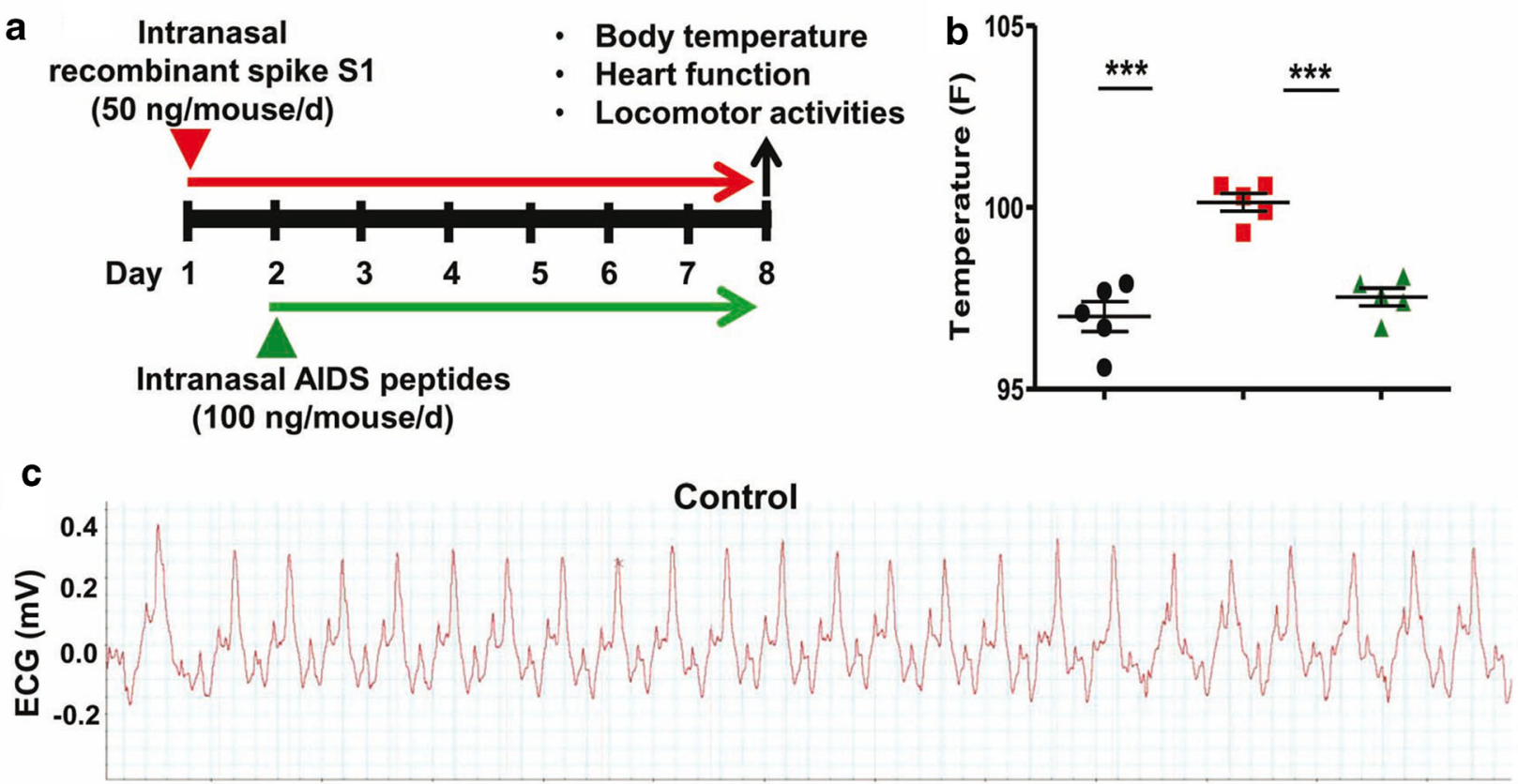

d

a

Intranasal
recombinant spike S1
(50 ng/mouse/d)

Intranasal AIDS peptides (100 ng/mouse/d)

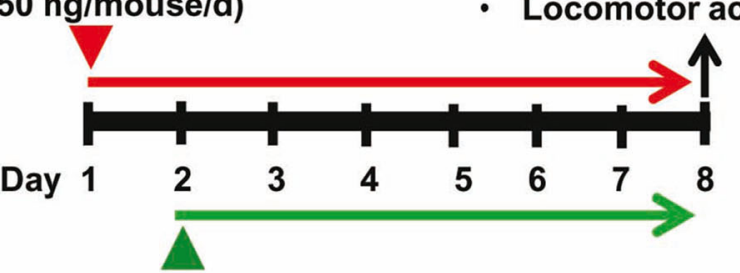

Spike S1

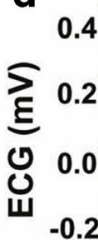

\section{4}

- Body temperature

- Heart function

- Heart function

0.2
0.0
-0.2

\section{e}

Spike S1 + wtAIDS

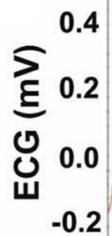

$-0.2$

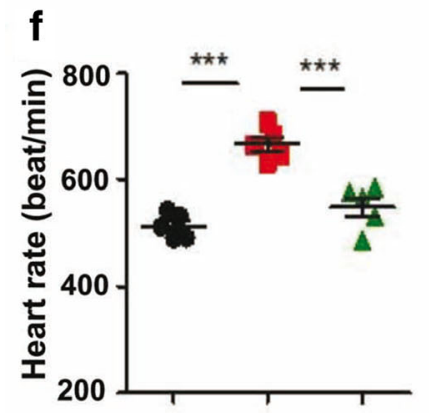

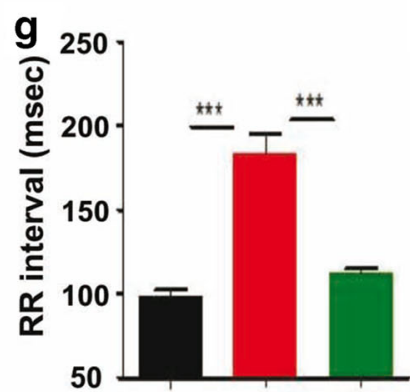

Control

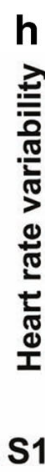

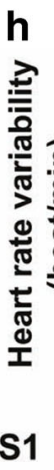

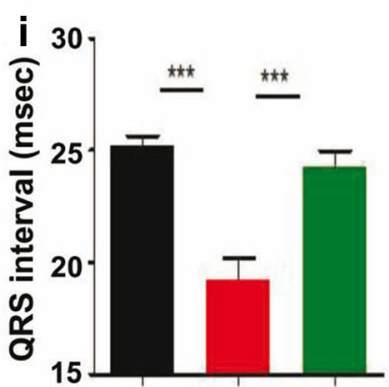

Spike S1 + wtAIDS
Fig. 5 Intranasal delivery of wtAIDS peptide suppresses disease progression in a mouse model of COVID-19. Six-eight week old C57/ BL6 mice $(n=5)$ of both sexes were treated intranasally with wtAIDS peptide (100 ng/mouse/d) from $1 \mathrm{~d}$ after intoxication of SARS-CoV-2 spike S1 (50 ng/mouse/d). After 7d of wtAIDS treatment, body temperature was measured followed by monitoring heart functions by non- invasive electrocardiography (ECG) using the PowerLab (ADInstruments) [a, schematic presentation of experiments; $\mathbf{b}$, body temperature; $\mathbf{c}$, chromatogram of control mice; d, chromatogram of spike S1intoxicated mice; e, chromatogram of (spike S1 + wtAIDS)-treated mice; f, heart rate; $\mathbf{g}$, RR interval; $\mathrm{H}$, heart rate variability; I, QRS interval]. ${ }^{* * * *} p<0.001$ 
Moreover, handling live SARS-CoV-2 has many biosafety requirements. Here, we describe the development of a very simple and live virus-free model of mimicking important cardiac and respiratory symptoms of COVID-19 in mice. SARS$\mathrm{CoV}-2$ spike $\mathrm{S} 1$ containing the RBD sequence is located at the $\mathrm{N}$-terminus of the spike protein. Since most of the neutralizing epitopes are located within the spike $\mathrm{S} 1$, this spike subunit has become an important candidate for vaccine development. Interestingly, here, we demonstrated that intranasal administration of the same spike S1 was capable of inducing many key features of COVID-19 (fever, lung inflammation, lung neutrophil infiltration, increase in serum IL-6, upregulation of serum CRP, and arrhythmias) in normal mice. Therefore, nasally SARS-CoV-2 spike S1-intoxicated mice could be used as a virus-free mouse model for evaluating different therapeutic options against COVID-19.

Until now, there is no effective therapy for COVID-19. Although vaccine will be soon available, COVID-19 might stay on the earth as a seasonal and an opportunistic event. For example, despite flu vaccination, about 40,000 to 50,000 people die each year in USA from flu. Therefore, a specific medicine for reducing SARS-CoV-2-related inflammatory events and taking care of respiratory and cardiac issues of COVID-19 will be necessary for better management of COVID-19 even in the postvaccine era. Although hydroxychloroquine showed some promise in the beginning, several clinical trials have ruled out the use of hydroxychloroquine in COVID-19 (Pahan and Pahan 2020). Only, Remdesivir has been approved for emergency use in COVID-19 (Lamb 2020). Although s.c. injection of some ICU patients with IFN $\beta$ - $1 \mathrm{~b}$ led to decrease in mortality rate (Rahmani et al. 2020), further randomized clinical trials with large sample size are needed for the exact estimation of survival benefit by this multiple sclerosis drug. Reduction of fever, decrease in serum IL6 and CRP, protection of lungs, normalization of heart functions, and improvement in locomotor activities in SARS-CoV-2 spike S1-intoxicated mice by intranasal treatment with wtAIDS peptide suggest that selective targeting of the SARS-CoV-2:ACE2 contact by wtAIDS peptide may be beneficial for COVID-19. The wtAIDS peptide should target only the SARS-CoV-2dependent ACE2 pathway without inhibiting normal physiological functions of ACE2. Accordingly, in a group of control mice, we also did not notice any drug-related side effect (e.g. hair loss, appetite loss, weight loss, untoward infection and irritation, etc.) upon treatment with intranasal wtAIDS peptide. In summary, our preclinical studies have identified intranasal AIDS peptide as a primary or adjunct therapeutic option for COVID-19 patients.

Supplementary Information The online version contains supplementary material available at https://doi.org/10.1007/s11481-020-09979-8.

Acknowledgements This study was supported by grants (AG050431, AT010980, and NS108025) from NIH to KP. Moreover, KP is the recipient of a Research Career Scientist Award (1IK6 BX004982) from the Department of Veterans Affairs.

\section{Compliance with Ethical Standards}

Conflict of Interest None.

\section{References}

Bao L et al (2020) The pathogenicity of SARS-CoV-2 in hACE2 transgenic mice. Nature 583:830-833

Chand S, Kapoor S, Orsi D, Fazzari MJ, Tanner TG, Umeh GC, Islam M, Dicpinigaitis PV (2020) COVID-19-associated critical illness-report of the first 300 patients admitted to intensive care units at a New York City Medical Center. J Intensive Care Med 35:963-970

Costela-Ruiz VJ, Illescas-Montes R, Puerta-Puerta JM, Ruiz C, Melguizo-Rodriguez L (2020) SARS-CoV-2 infection: The role of cytokines in COVID-19 disease. Cytokine Growth Factor Rev 54: $62-75$

Du L, He Y, Zhou Y, Liu S, Zheng BJ, Jiang S (2009) The spike protein of SARS-CoV-a target for vaccine and therapeutic development. Nat Rev Microbiol 7:226-236

Edwards MR, Bartlett NW, Hussell T, Openshaw P, Johnston SL (2012) The microbiology of asthma. Nat Rev Microbiol 10:459-471

Ghosh A, Roy A, Liu X, Kordower JH, Mufson EJ, Hartley DM, Ghosh S, Mosley RL, Gendelman HE, Pahan K (2007) Selective inhibition of NF-kappaB activation prevents dopaminergic neuronal loss in a mouse model of Parkinson's disease. Proc Natl Acad Sci U S A 104: 18754-18759

Gordon JW, Shaw JA, Kirshenbaum LA (2011) Multiple facets of NFkappaB in the heart: to be or not to NF-kappaB. Circ Res 108:11221132

$\mathrm{Gu} \mathrm{H}$ et al (2020) Adaptation of SARS-CoV-2 in BALB/c mice for testing vaccine efficacy. Science 369:1603-1607

Jana M, Jana A, Liu X, Ghosh S, Pahan K (2007) Involvement of phosphatidylinositol 3-kinase-mediated up-regulation of I kappa B alpha in anti-inflammatory effect of gemfibrozil in microglia. J Immunol 179:4142-4152

Jiang F, Yang J, Zhang Y, Dong M, Wang S, Zhang Q, Liu FF, Zhang K, Zhang C (2014) Angiotensin-converting enzyme 2 and angiotensin 1-7: novel therapeutic targets. Nat Rev Cardiol 11:413-426

Karamchandani K, Quintili A, Landis T, Bose S (2020) Cardiac arrhythmias in critically Ill patients with COVID-19: a brief review. J Cardiothorac Vasc Anesth

Lamb YN (2020) Remdesivir: First Approval. Drugs 80:1355-1363

Ledford H (2020) How does COVID-19 kill? Uncertainty is hampering doctors' ability to choose treatments. Nature 580:311-312

Machhi J, Herskovitz J, Senan AM, Dutta D, Nath B, Oleynikov MD, Blomberg WR, Meigs DD, Hasan M, Patel M, Kline P, Chang RC, Chang L, Gendelman HE, Kevadiya BD (2020) The natural history, pathobiology, and clinical manifestations of SARS-CoV-2 infections. J Neuroimmune Pharmacol 15:359-386

Matute-Bello G, Downey G, Moore BB, Groshong SD, Matthay MA, Slutsky AS, Kuebler WM, Acute Lung Injury in Animals Study G (2011) An official American Thoracic Society workshop report: features and measurements of experimental acute lung injury in animals. Am J Respir Cell Mol Biol 44:725-738

McCullers JA (2014) The co-pathogenesis of influenza viruses with bacteria in the lung. Nat Rev Microbiol 12:252-262

Mehta P, McAuley DF, Brown M, Sanchez E, Tattersall RS, Manson JJ, Hlh Across Speciality Collaboration UK (2020) COVID-19: consider cytokine storm syndromes and immunosuppression. Lancet 395:1033-1034

Mondal S, Jana M, Dasarathi S, Roy A, Pahan K (2018) Aspirin ameliorates experimental autoimmune encephalomyelitis through interleukin-11mediated protection of regulatory T cells. Sci Signal 11:eaar8278 
Mondal S, Kundu M, Jana M, Roy A, Rangasamy SB, Modi KK, Wallace J, Albalawi YA, Balabanov R, Pahan K (2020) IL-12 p40 monomer is different from other IL-12 family members to selectively inhibit IL-12Rbeta1 internalization and suppress EAE. Proc Natl Acad Sci U S A 117:21557-21567

Pahan P, Pahan K (2020) Smooth or risky revisit of an old malaria drug for COVID-19? J Neuroimmune Pharmacol 15:174-180

Pahan K, Sheikh FG, Liu X, Hilger S, McKinney M, Petro TM (2001) Induction of nitric-oxide synthase and activation of NF-kappaB by interleukin-12 p40 in microglial cells. J Biol Chem 276:7899-7905

Pia L (2020) Spatial resolution of SARS-CoV-2 lung infection. Nat Rev Immunol

Rahmani H, Davoudi-Monfared E, Nourian A, Khalili H, Hajizadeh N, Jalalabadi NZ, Fazeli MR, Ghazaeian M, Yekaninejad MS (2020) Interferon beta-1b in treatment of severe COVID-19: A randomized clinical trial. Int Immunopharmacol 88:106903

Rangasamy SB, Dasarathi S, Pahan P, Jana M, Pahan K (2019) Low-dose aspirin upregulates tyrosine hydroxylase and increases dopamine production in dopaminergic neurons: implications for Parkinson's disease. J Neuroimmune Pharmacol 14:173-187

Rangasamy SB, Jana M, Roy A, Corbett GT, Kundu M, Chandra S, Mondal S, Dasarathi S, Mufson EJ, Mishra RK, Luan CH, Bennett DA, Pahan K (2018b) Selective disruption of TLR2MyD88 interaction inhibits inflammation and attenuates Alzheimer's pathology. J Clin Invest 128:4297-4312
Roy A, Jana M, Corbett GT, Ramaswamy S, Kordower JH, Gonzalez FJ, Pahan K (2013) Regulation of cyclic AMP response element binding and hippocampal plasticity-related genes by peroxisome proliferator-activated receptor alpha. Cell Rep 4:724-737

Roy A, Jana M, Kundu M, Corbett GT, Rangaswamy SB, Mishra RK, Luan CH, Gonzalez FJ, Pahan K (2015) HMG-CoA reductase inhibitors bind to PPARalpha to upregulate neurotrophin expression in the brain and improve memory in mice. Cell Metab 22:253-265

Sahu BR, Kampa RK, Padhi A, Panda AK (2020) C-reactive protein: A promising biomarker for poor prognosis in COVID-19 infection. Clin Chim Acta 509:91-94

Stower H (2020) Spread of SARS-CoV-2. Nat Med 26:465

Vickers C, Hales P, Kaushik V, Dick L, Gavin J, Tang J, Godbout K, Parsons T, Baronas E, Hsieh F, Acton S, Patane M, Nichols A, Tummino P (2002) Hydrolysis of biological peptides by human angiotensin-converting enzyme-related carboxypeptidase. J Biol Chem 277:14838-14843

Zaman MA, Oparil S, Calhoun DA (2002) Drugs targeting the reninangiotensin-aldosterone system. Nat Rev Drug Discov 1:621-636

Publisher's Note Springer Nature remains neutral with regard to jurisdictional claims in published maps and institutional affiliations. 